\title{
Cross-reactivity pattern of a rare presentation of generalized delayed-type hypersensitivity to local anaesthetics
}

\author{
Jaime S Rosa Duque, Patrick CY Chong, Yu-Lung Lau, Marco HK Ho
}

\begin{abstract}
A seven-year-old girl developed angioedema and a generalized, erythematous rash several hours after receiving lignocaine with adrenaline reproducible on provocative challenge, confirming the first known case of generalized delayed-type hypersensitivity to local anaesthetics with cross-reactivity to bupivacaine but not chloroprocaine.
\end{abstract}

\section{From:}

Department of Paediatrics and Adolescent Medicine, Queen Mary Hospital,

Li Ka Shing Faculty of Medicine, The University of Hong Kong, Hong Kong.

\section{Corresponding author:}

Jaime S Rosa Duque

Clinical Assistant Professor of Department of Paediatrics and Adolescent Medicine

Honorary Tutor of Department of Medicine

Room 115, New Clinical Bldg

Queen Mary Hospital, 102 Pokfulam Road

E-mail: jsrduque@hku.hk

\section{Introduction}

T-cell-mediated delayed-onset contact dermatitis (CD) is more common with topical exposures to ester, as opposed to, amide local anaesthetics (LAs) and infrequently seen after parenteral administration. ${ }^{1,2}$ Immunoglobulin-E (IgE)-mediated acute-onset allergy to LAs seldom occurs, but IgE-mediated cross-reactivity between lidocaine and mepivacaine has been reported. ${ }^{1-3}$ Here we present a rare case of a paediatric patient with generalized delayed-type hypersensitivity (DTH) due to LAs and describe the cross-reactivity between LAs for this type of drug hypersensitivity which is not well known. ${ }^{4}$

Prior to a dental restoration procedure, a seven-year-old girl received $1.7 \mathrm{~mL}$ of $2 \%$ lignocaine (an amide $\mathrm{LA}$ ) with 1:80,000 adrenaline (full list of diluents and excipients not available) via buccal infiltration. Two to three hours later, the parents noticed swelling of her face and eyelids; later that night, she developed a pruritic, erythematous rash on her arms and face that spontaneously resolved one week later. There was no other symptom such as urticaria, wheeze, shortness of breath, gastrointestinal complaints, neurologic changes, or cardiovascular compromise. Aside from chronic mild dry skin, she had no history of atopy or exposure to other new medications, foods, or environmental allergens. The patient tolerated LAs in the past, also as part of her dental treatments, but the parents were not certain which specific LAs she had received.

The patient was referred to our paediatric allergy service for evaluation of whether she had an allergy to lignocaine and whether there was a viable alternative such that the patient could avoid general anaesthesia for simple dental procedures.
Skin tests using epicutaneous prick, intradermal injection, atopy topical patch for 48-72 hours, and a subcutaneous provocative challenge injected into her right arm with lignocaine (containing methylparaben and other preservatives) and adrenaline were initially negative (Figure 1). However, one day after the visit, she developed several non-urticarial, non -vesicular, non-pustular, pruritic, patchy, flat, blanchable, erythematous lesions located at and scattered distal to the sites of injection including the skin overlying her right medial malleolus (Figure 2).

Since amide LAs have the potential to not share cross -reactivity according to previous observations for $\mathrm{CD}$, and bupivacaine (another amide LA) was the only other local anaesthetic available under formulary in Hong Kong, the patient underwent skin testing and subcutaneous challenge to preservative-free (not containing methylparaben) $0.5 \%$ bupivacaine. Again, she demonstrated a lack of localized skin reactivity to preservative-free $0.5 \%$ bupivacaine immediately after epicutaneous prick, intradermal injection, and subcutaneous provocative challenge. Unfortunately, the patient experienced a similar generalized rash to bupivacaine (albeit occurring at other skin areas), confirming the patient's hypersensitivity to local anaesthetics.

As such, a non-formulary medication request was arranged to import preservative-free $2 \%$ chloroprocaine in hopes that the patient's drug hypersensitivity does not share cross-reactivity with this ester LA. Subsequently, the patient had no reaction to atopy patch and subcutaneous provocation testing to chloroprocaine. 


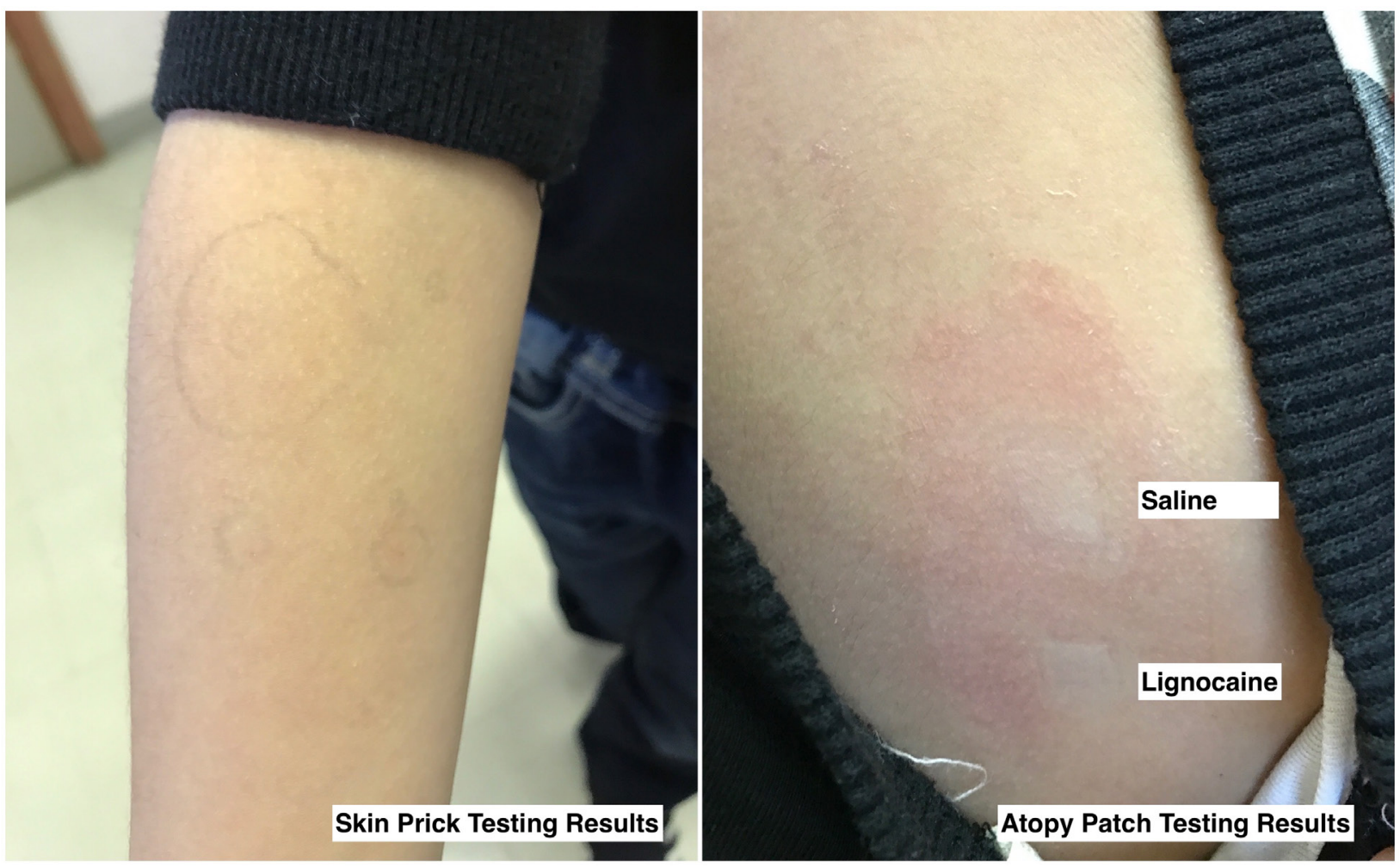

Figure 1. Negative immediate-reading epicutaneous prick and intradermal skin test, and negative 48 hours-reading of patch test to lignocaine with adrenaline (excipients: methylhydroxybenzoate/methylparaben, propylhydroxybenzoate, sodium chloride, potassium metabisulphite, disodium edentate, sodium hydroxide solution, water).
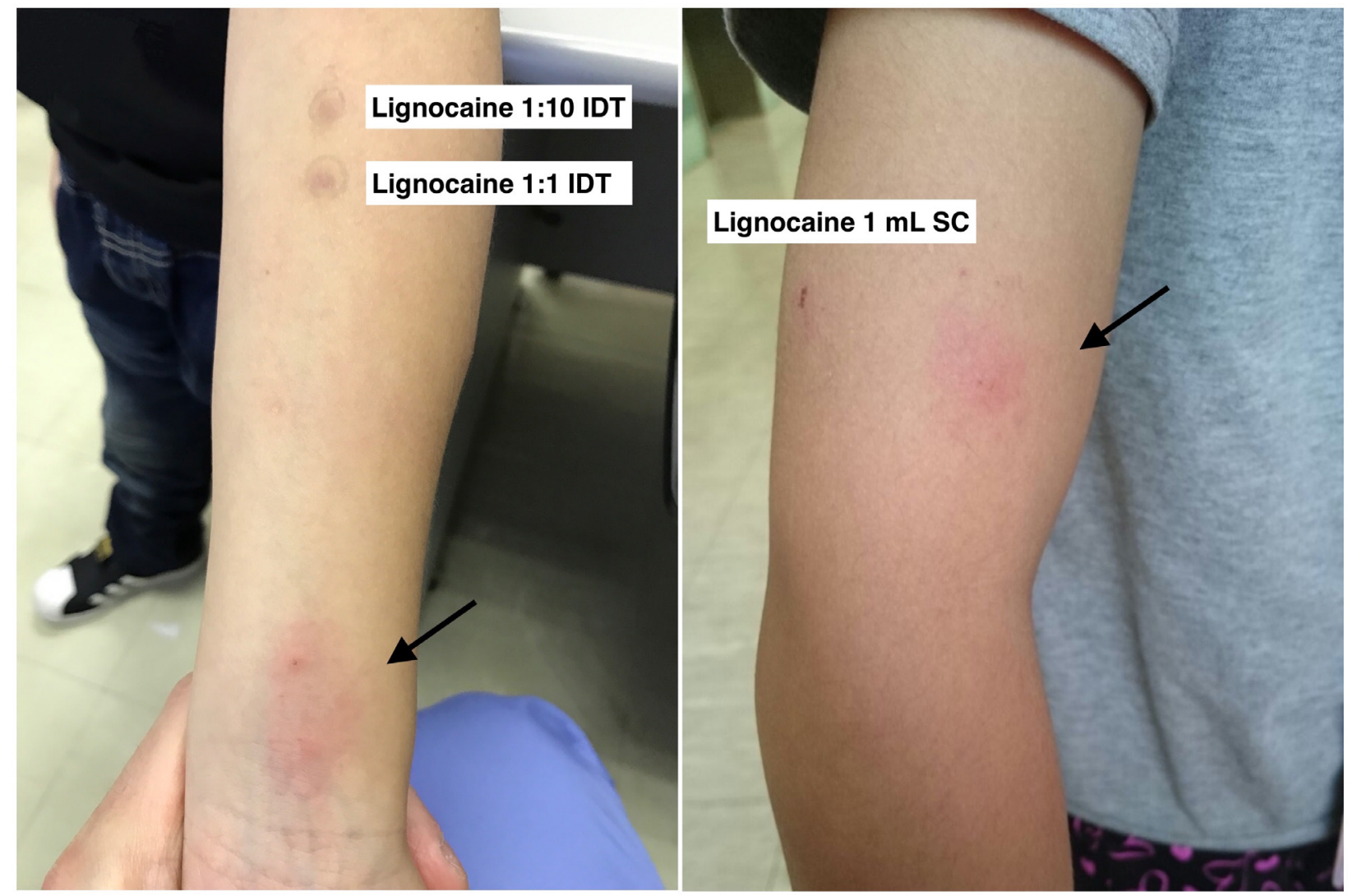

Figure 2. Diffuse erythematous, pruritic, non-urticarial rash located at and distal to sites of injection one day after skin and subcutaneous provocation testing. 

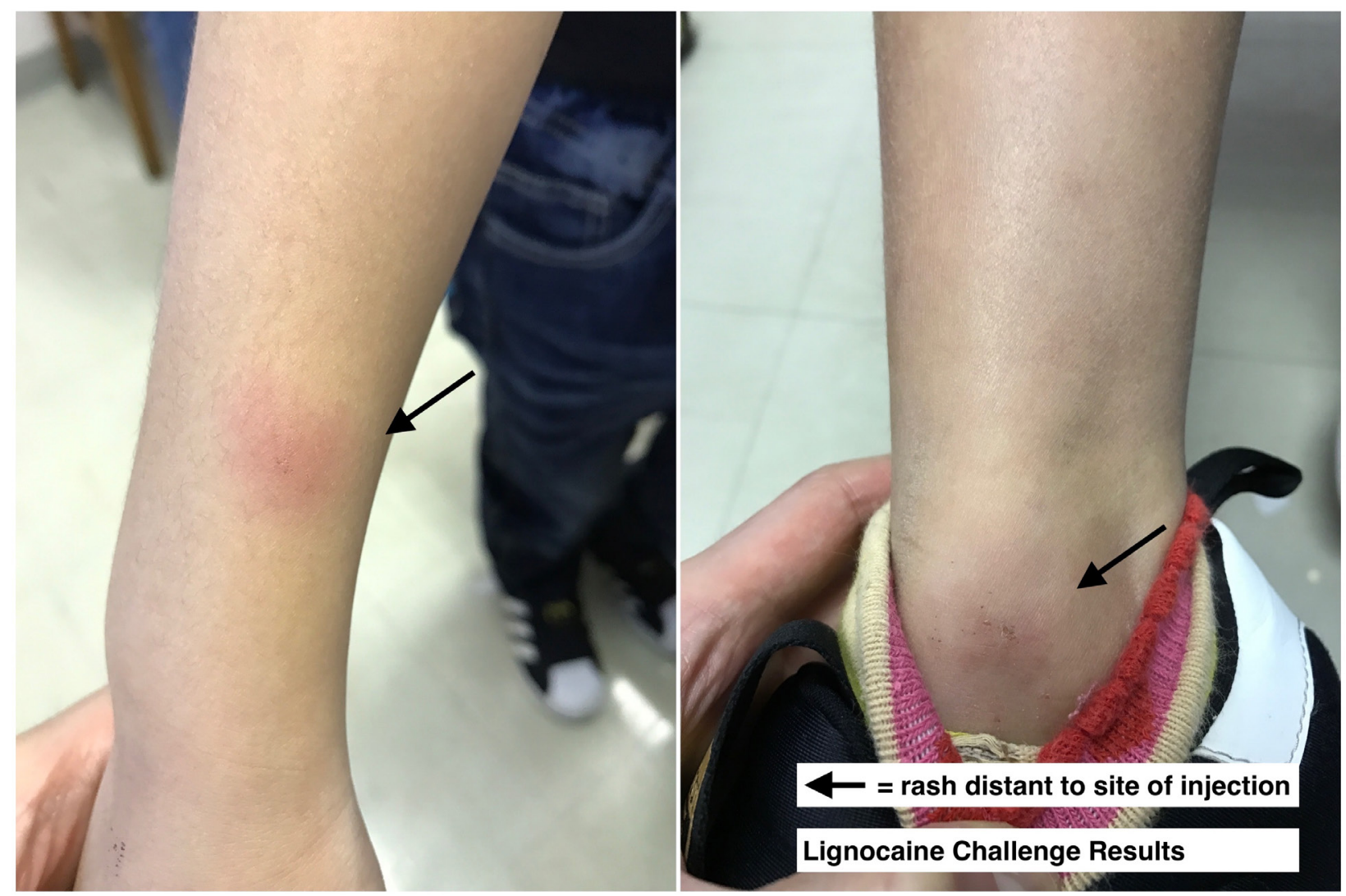

Figure 2. (Continued)

\section{Discussion}

Most adverse reactions to LAs are due to non-allergic factors that include vasovagal responses, anxiety and panic attacks, and pharmacologic effects such as hypoesthesia, methemoglobinemia, and dysrhythmias, with the risk of the latter increased if the medication is inadvertently administered intravenously alongside adrenaline. ${ }^{1}$ Immunologic hypersensitivity to LA is estimated to be $\leq 1 \%$, most of which are due to cell-mediated CD to the LA or the associated excipients. ${ }^{1,5}$ Patients who often receive treatments under anaesthesia and healthcare professionals are more likely to become sensitized from frequent but inconsistent exposures.

Immediate-type reactions, urticarial vasculitis, fixed drug eruption, and acute generalized exanthematous pustulosis (AGEP) are even more rare. ${ }^{1,5-8}$ However, to minimize the risk of life-threatening recurrences, if adverse events arise after LA administration, obtaining a thorough history and performing skin and provocation testing would be a reasonable approach. ${ }^{1,2}$ The rates of false-positive and false-negative results for skin testing to LA are not known precisely since standardized non-irritating concentrations with validated predictive values for clinical allergy have not been systematically characterized. ${ }^{1,2}$ Allergy to methylparabens and other additives has also been documented., ${ }^{1,2}$ Therefore, provocative challenge to the preservative-free formulation without adrenaline remains the gold-standard diagnostic test for LA hypersensitivity. ${ }^{4,69,10}$ The reason that adrenaline and preservatives alongside lignocaine were used initially for testing was to ascertain whether the patient truly had a reproducible hypersensitivity to the compounds contained in the injection versus the possibility of an inconsistent irritant effect or merely an exacerbation of an underlying, low-grade atopic eczema from the dental procedure or other triggers. ${ }^{4}$ Since this first step was positive, the patient was deemed allergic to either the medication or excipients (which may also be contained in other medications or cosmetic mixtures). In the next step, when the patient also reacted to bupivacaine without adrenaline or preservatives, it confirmed her hypersensitivity to amide LAs.

If persistence of drug hypersensitivity to the LA is confirmed, such as in our patient's case, identification of a non-cross-reactive alternative LA can be pursued. ${ }^{1,2}$ The allergenicity pattern between LAs for CD may be related to the chemical structure group-group 1 benzoic acid ester (e.g., chloroprocaine) and group 2 amides (e.g., lignocaine, bupivacaine) - that is bonded to the lipophilic, aromatic residue. ${ }^{1,2}$ On the basis of atopy patch testing, amide derivatives are less likely to cross-react with each other or with benzoic acid ester LAs, but cross-reactivity within the same ester group is more typical since many ester LAs are converted in vivo to a common metabolite and potential allergen: para-aminobenzoic acid. ${ }^{2}$ It is unknown if this same pattern is relevant for IgE-mediated and other types of immunologically-mediated reactions, which have only been reported in the literature as sporadic cases rather than investigated under large-scale, prospective, controlled trials. ${ }^{11}$

As cross-reaction with allergens (such as foods) other than LAs has not been reported, it is likely that our patient was sensitized to the amide LAs due to exposures from previous 
procedures after anaesthesia or via topically applied LAs that are commonly mixed with other medications such as antimicrobial ointments for wounds. ${ }^{1,2}$ She did not undergo skin biopsy or laboratory testing to confirm her specific type of hypersensitivity as the cutaneous eruption was self-limiting and the patient preferred to avoid unnecessary invasive tests. The clinical diagnosis of DTH was established based on the morphologic appearance, distribution, and onset time and duration of the rash, which were most consistent with DTH rather than other reactions such as symmetrical drug-related intertriginous and flexural exanthema, other vesicular types of systemic CD, fixed drug eruption, AGEP, drug reaction with eosinophilia and systemic symptoms, or Steven-Johnson syndrome. ${ }^{1}$

Although rarely reported, reproducible generalized DTH to amide LAs can occur. Cross-reactivity was not observed when an ester LA was administered, allowing this patient to be able to avoid the need to receive general anesthesia for minor invasive procedures in the future.

\section{References}

1. Joint Task Force on Practice Parameters, American Academy of Allergy, Asthma and Immunology, American College of Allergy, Asthma and Immunology, Joint Council of Allergy, Asthma and Immunology. Drug allergy: an updated practice parameter. Ann Allergy Asthma Immunol. 2010;105:259-73.
2. Thyssen JP, Menne T, Elberling J, Plaschke P, Johansen JD. Hypersensitivity to local anaesthetics--update and proposal of evaluation algorithm. Contact Dermatitis. 2008;59:69-78.

3. Fuzier R, Lapeyre-Mestre M, Mertes PM, Nicolas JF, Benoit Y, Didier A, et al. Immediate- and delayed-type allergic reactions to amide local anesthetics: clinical features and skin testing. Pharmacoepidemiol Drug Saf. 2009;18: 595-601.

4. Bircher AJ, Messmer SL, Surber C, Rufli T. Delayed-type hypersensitivity to subcutaneous lidocaine with tolerance to articaine: confirmation by in vivo and in vitro tests. Contact Dermatitis. 1996;34:387-9.

5. Bhole MV, Manson AL, Seneviratne SL, Misbah SA. IgE-mediated allergy to local anaesthetics: separating fact from perception: a UK perspective. $\mathrm{Br}$ J Anaesth. 2012;108:903-11.

6. Vega F, Argiz L, Bazire R, Las Heras P, Blanco C. Delayed urticaria due to bupivacaine: A new presentation of local anesthetic allergy. Allergol Int. 2016;65:498-500

7. Torres MJ, Garcia JJ, del Cano Moratinos AM, Rondon C, Blanca M Fixed drug eruption induced by mepivacaine. J Allergy Clin Immunol. 1995;96:130-1.

8. O’Toole A, Lacroix J, Pratt M, Beecker J. Acute generalized exanthematous pustulosis associated with 2 common medications: hydroxyzine and benzocaine. J Am Acad Dermatol. 2014;71:e147-9.

9. Corbo MD, Weber E, DeKoven J. Lidocaine Allergy: Do Positive Patch Results Restrict Future Use? Dermatitis. 2016;27:68-71.

10. Tomoyasu Y, Mukae K, Suda M, Hayashi T, Ishii M, Sakaguchi M, et al. Allergic reactions to local anesthetics in dental patients: analysis of intracutaneous and challenge tests. Open Dent J. 2011;5:146-9.

11. Sharma V, Harper NJ, Garcez T, Arkwright PD. Allergic reaction to mepivacaine in a child. Br J Anaesth. 2013;110:1059-60. 